\title{
Using administrative data held by DWP as an alternative sampling frame for the Family Resources Survey
}

\author{
Antonia Simon \\ Department for Work and Pensions
}

\section{Summary}

The Family Resources Survey (FRS) is the leading UK household survey for the collection of private household income. The Department for Work and Pensions (DWP) is currently considering the extent to which their administrative data can be used to develop an alternative sampling frame for the FRS. This article summarises the major findings of this research, comparing the current sampling frame for the FRS, using the small-users Postcode Address File (PAF), with suitable administrative data sources. The findings offer important considerations for how UK household surveys might be sampled in the future using administrative data sources.

\section{Background}

The use of administrative data for statistical research purposes has been of growing interest in the UK (Jenkins et al 2007). There are a number of perceived benefits with administrative data, including a reduction in costs associated with conducting large-scale surveys and an improvement in data quality. The use of administrative data may also help to diminish respondent burden and the public opposition to completing questionnaires, including the census (Calderwood and Lessof 2006; Jones and Elias 2006; Wroe 1998). However, relative to other countries, such as Finland and Denmark, the UK has so far made little use of administrative data as a sampling frame for surveys.

Recognising the wider potential benefits of better utilising its administrative data, the Department for Work and Pensions (DWP) is considering the extent to which such data can be used for sampling purposes. To investigate this further, the DWP commissioned research to investigate the feasibility of using DWP administrative data as an alternative sampling frame for the Family Resources Survey (FRS). This research compared the current sampling frame for the FRS with two main DWP administrative data sources: the Address Hierarchy (AH) File, which forms the address file for the Work and Pensions Longitudinal Study (WPLS), and the Customer Information 
System (CIS) which represents a more complete list of all individuals who have been allocated a National Insurance Number (NINO). The AH file contains an address history for individuals who have had some contact with DWP through claiming benefits or their state pension. It excludes individuals not known to the DWP. The CIS, in contrast, includes all NINOs and therefore potentially covers all of the population aged 16 years and over (and not just DWP customers). For the analysis reported in this paper, the $\mathrm{AH}$ file was reduced to represent one address per household - with only current addresses included (assumed to be the last registered address per individual).

\section{Findings}

\section{The FRS and its current sampling frame}

The FRS has collected household income data at a national level in its current form since 1993/4. It achieves an interviewed sample size of approximately 25,000 households a year. FRS data are used to assess progress towards achieving key Departmental Objectives to reduce income poverty among household types identified to be at risk, including pensioners and people with disabilities. The FRS, along with many other national household surveys in the UK, uses the small-users Postcode Address File (PAF) as the sampling frame for Great Britain (GB). This is now the most commonly used sampling frame for large-scale surveys of the general population (Tipping and Nicolaas 2006). Compiled by Royal Mail, this is a list of all addresses in the UK receiving fewer than 50 Items of post daily. While the PAF provides full address detail, it does not include any further information that might be useful for sampling, such as household composition or age structure of households.

The contractors for the FRS (currently the National Centre for Social Research and the Office for National Statistics) aim to draw a statistically representative sample from the PAF using a combination of sampling techniques such as stratification based on the latest census, systematic randomisation, and clustering (ONS and NatCen 2007). The selection process also ensures a particular household is not oversampled so as to avoid complaints from members of the public being approached more than once within a short time period to participate in government surveys (Wilson and Elliott, 1987). In addition, any multi-households in the sample are treated to take account of the differing probabilities of selection this introduces (ONS and NatCen 2007).

\section{The geographical coverage of DWP administrative data relative to the PAF}

Postcodes, and then addresses from the PAF were matched separately to the $\mathrm{AH}$ file and the $\mathrm{CIS}$. As part of the matching process, postcodes were validated using British Standards 7666 'BS 7666', which describes postcodes as being 'valid' if they are alphanumeric and between five and eight characters long (Cabinet Office, 2009). There are just under 1.5 million unique postcodes recorded on the small-users PAF, just over 1.7 million unique postcodes on the $\mathrm{CIS}$, and just over 1.8 million unique postcodes on the $\mathrm{AH}$ file. The $\mathrm{AH}$ file has a larger number of postcodes than the $\mathrm{CIS}$ because it includes a historical record of addresses based on an individual's history of claiming a 
state benefit or state pension through the Department. Therefore, it is common to identify more than one address per individual in this file.

For postcodes, the 'match rate' for the PAF to the CIS and AH files was quite high - with 98 per cent of PAF's postcodes found on the CIS and 97 per cent found on the AH file (Table 1). For addresses, the match rate of the PAF to the CIS was 84 percent and to the AH file was 75 per cent. The match rate for addresses was lower than for postcodes because it was not possible to match addresses without street numbers (for example, those starting with a house name like 'Rose Cottage'). The lower match rate for addresses also reflects the less reliable quality of addresses in the administrative data. For example, addresses on the CIS and AH files are more likely to have errors and /or different formats. The most common issues are:

- information for flats being dropped

- suffixes on addresses being dropped (for example 41B becomes 41)

- flat and house numbers becoming confused (for example flat 4, 7 street becomes flat 7, 7 street); and

- addresses have no street / house number

The match rate for addresses for the AH file was much lower than for the CIS (75 per cent compared with 84 per cent for the CIS). This reflects the lower geographical coverage of the AH file relative to the CIS. While the $\mathrm{CIS}$ and $\mathrm{AH}$ files contain a similar number of higher level unique postcodes, the CIS offers greater coverage of lower level addresses within each unique postcode. There was no geographical bias in the match-rate for postcodes or addresses, in that the match rate was fairly similar across postcode letters on the CIS (ranging from 83 per cent to 90 per cent). The exception to this pattern was addresses with 'B' postcodes, with fewer matching to the CIS and AH files because 'B' postcodes include Belfast (but actually cover all of Northern Ireland). Those 'B' postcodes which cover Belfast (and Northern Ireland) are excluded from the PAF frame for the FRS.

\section{Table $1 \quad$ Matching $\mathrm{CIS}$ and $\mathrm{AH}$ administrative data by postcode to the small-users PAF}

Percentage of postcodes on PAF matched to $\mathrm{CIS}$ and $\mathrm{AH}$ file

\begin{tabular}{lcc}
\hline & CIS & AH file on WPLS \\
\hline PAF unique postcodes matched & $98.3 \%$ & $97.7 \%$ \\
& $(1,446,849)$ & $(1,439,262)$ \\
PAF unique postcodes not matched & $1.7 \%$ & $2.21 \%$ \\
& $(24,972)$ & $(32,559)$ \\
Total unique postcodes on PAF & $100 \%$ & $100 \%$ \\
\end{tabular}




\section{Analysing the extent of match between the PAF and administrative sources}

While we would expect most CIS postcodes to find a match on the PAF, approximately 330,000 failed to do so. Of these, 90 per cent $(300,000)$ had 'valid' Royal Mail (RM) postcode combinations (Table 1), as verified using the British Standards by which postcode formats are judged ('BS 7666': see previous section). Three reasons were hypothesised to explain why some postcodes from the CIS did not find matches on the PAF:

- the postcodes could belong to a deceased or 'dead letter office' (DLO) flagged individual - such a flag given where post is returned undelivered, or information is otherwise received that the person does not live at that address, or

- to a person with a foreign address (representing individuals now living abroad who for some reason had been allocated a NINO on the CIS), or

- be deleted postcodes no longer in use by Royal Mail (RM) because they may have belonged to a now demolished building

An investigation of the cases on the CIS that did not match the PAF, showed approximately half of these postcodes did not match the PAF because they were from 'old' addresses. These old addresses were flagged in the administrative data as deceased or DLO flagged. While this status is not in itself a problem for use in a sampling frame, so long as the address itself is valid, these addresses failed to find a match in the PAF because either they had been entered incorrectly in the administrative data or were addresses/postcodes that were no longer in use by Royal Mail. In addition, only a small minority ( 0.5 percent) of all cases not matched to PAF were found to be foreign addresses.

The 'valid, non-match' postcodes between the CIS and PAF were also compared to the National Statistics Postcode Directory (NSPD). The NSPD is an ONS database which records all postcodes that are currently, or have in the past, been in Royal Mail circulation. A 'date deleted' flag in the NSPD was used to indicate whether postcodes have been deleted. Approximately 31 per cent of these ('valid non-match') postcodes were found to have been deleted (see Table 2). The rest of these cases were 'dormant' (32 per cent), describing postcodes formerly in circulation but which are not currently in use (but not flagged as deleted), or 'large-users' (37 per cent) - 'live' or 'current' postcodes but excluded from the small-users PAF as they belong to large (expected to be business) addresses.

\section{Table 2 Reasons why postcodes with 'valid' RM formats on CIS do not always match the small-users PAF}

\begin{tabular}{lc}
\hline $\begin{array}{l}\text { Reason for 'valid' non-matched cases between CIS and PAF and between small-users } \\
\text { PAF and NSPD }\end{array}$ & $\begin{array}{c}\text { \% non-matched } \\
\text { postcodes }\end{array}$ \\
\hline Deleted postcodes: found on CIS but flagged as deleted on NSPD & $31 \%$ \\
Dormant postcodes: found on CIS, not flagged on NSPD as deleted but not identified as 'live' & $32 \%$ \\
Large user postcodes: found on CIS and identified as 'live' on NSPD & $37 \%$ \\
Total on CIS not matching PAF but with 'valid' postcode format & $100 \%$ \\
\end{tabular}


Although Table 2 shows the reasons for 'valid non-match' postcodes were overall fairly evenly distributed between 'deleted', 'dormant' and 'large-user', there was actually some variation in these proportions when examined by postcode letter (for example the first letter in the postcode SW10 2PP would examine the 'S's). For example, 71 per cent of 'A's were identified as not matching and 'dormant' on the NSPD, letters ' $\mathrm{B}$ ', 'l', and ' $\mathrm{J}$ ' had high proportions not matching and 'large users' (71 per cent, 59 per cent and 96 per cent respectively), and letters ' $Z$ ', ' $T$ ', E' and 'F' had high proportions not matching and 'deleted' (60 per cent, 45 per cent, $46 \%$ per cent and 44 per cent respectively).

\section{Technical considerations with using administrative data for sampling}

While it is always important to consider ethical impacts, there are likely to be few ethical concerns posed if administrative data are used to generate an address list for statistical sampling purposes. This is because the information generated for a sampling frame would approximate (in terms of level of detail) to that available in the PAF and which is currently widely used for sampling purposes. However, there are a number of key technical considerations in creating a reliable FRS sampling frame, including:

- reliably dealing with addresses that do not match because of data quality - work is currently underway elsewhere in DWP to 'clean' administrative data such as the CIS so that it holds more complete and reliable data and better matches the full PAF

- reducing administrative data to unique addresses so that each represents a household currently administrative data, such as the $\mathrm{AH}$ file can hold several records per individual according to their benefit or pension claim histories

- excluding foreign addresses and GB addresses which do not represent private households

\section{Conclusions}

This article has outlined the potential for using administrative sources for developing alternative sources of sampling frames for the FRS. It has investigated the geographical coverage of administrative data relative to the current sampling frame for the FRS, using the small-users PAF.

The findings discussed here indicate that administrative data have the potential to provide a good alternative for sampling the FRS. This is because the match of PAF postcodes to the administrative data examined in this research is quite high (possibly even higher when non-private households are excluded from the $\mathrm{CIS}$ and $\mathrm{AH}$ files). The match rate of addresses, particularly using the CIS, also seems to offer good coverage of the GB population. However, further work is needed to make using administrative data as an alternative sampling frame for the FRS a viable option. This includes cleaning addresses on the CIS to improve the data quality and carrying out a full assessment of any bias. For example, unacceptable bias may be introduced if all addresses without house numbers or with house names are never included in a sampling frame.

While this research has concentrated on comparing the CIS and AH files with the FRS, they demonstrate the real potential of using administrative data for developing sampling frames for 
other household surveys, such as the Labour Force Survey. This would also tally with wider national initiatives to make better use of administrative data held across government.

\section{Acknowledgements}

This article derives from a project jointly funded by the DWP and the Economic Social Research Council (grant number: RES-173-27-0204). The author is very grateful to John Snow, at the DWP, for his technical guidance and support with using and analysing DWP administrative data; also for the support given with this research from Valerie Christian, at the DWP, and Charlie Owen, at the Institute of Education (IOE). Thanks also to the IOE ethics committee for reviewing the work reported here and for granting full ethical approval for this research.

\section{Further information}

Further details about the Family Resources Survey can be obtained from the DWP's website: http://research.dwp.gov.uk/asd/frs/

\section{Contact}

The author of this paper, Antonia Simon, is an academic researcher at the Institute of Education, University of London. She was seconded to the DWP (February 2010 to March 2011) to carry out the research reported in this paper. Further information about this work can be obtained from her at: a.simon@ioe.ac.uk or the team manager at the DWP, Valerie Christian at: Valerie.Christian@dwp.gsi.gov.uk.

\section{References}

Cabinet Office (2009) UK postcode data formats. Retrieved from Cabinet Office: http://interim.cabinetoffice.gov.uk/govtalk/schemasstandards/egif/datastandards/address/postcode.aspx

Calderwood L and Lessof C (2006) 'Enhancing longitudinal surveys by linking administrative data'. Paper presented at the MOLS2006 Conference, 12-14 July 2006, University of Essex

Jenkins S P, Lynn P, Jäckle A, and Sala E (2007) 'The feasibility of linking household survey and administrative record data: new evidence for Britain', International Journal of Social Research Methodology, 11(1), 29-43

Jones P, and Elias P (2006) Administrative data as a research resource: A selected audit. ESRC National Centre for Research Methods (Unpublished). Retrieved from: http://eprints.ncrm.ac.uk/452/1/Admin\%2520Data\%2520selected\%2520audit\%2520Dec06.pdf

Office for National Statistics \& National Centre for Social Research (2007). Family Resources Survey. Annual technical report 2007-8. Prepared for Department of Work and Pensions 
Tipping S and Nicolaas G (2006) 'In search of a population sampling frame for UK postal surveys', Survey Methodology Bulletin, 58: August 2006

Wilson P R and Elliott D J (1987) 'An evaluation of the Postcode Address File as a sampling frame and its use within OPCS', Journal of the Royal Statistical Society A, 150 part 3: 230-240

Wroe D (1998) Beyond 2001. Alternative to the Census. Study for Office for National Statistics. Volume 1: Report. Office for National Statistics, Crown Copyright. Retrieved from: www.statistics.gov.uk/downloads/theme_population/previous_uk.pdf 